\title{
Electronic Sensor Prototype to Detect and Measure Curvature Through Their Curvature Energy
}

\author{
Francisco Bulnes ${ }^{1}$, Isaías Martínez ${ }^{2}$, Omar Zamudio ${ }^{3}$, Gabriel Negrete ${ }^{4}$ \\ ${ }^{1}$ Research Department in Mathematics and Engineering, TESCHA, and GI, Chalco, Mexico \\ ${ }^{2}$ Electronic Engineering Division TESCHA and GI, Chalco, Mexico \\ ${ }^{3}$ Electronic Engineering Division TESCHA, Chalco, and GI, Chalco, Mexico \\ ${ }^{4}$ Electronic Engineering Division GI-TESCHA, Chalco, and GI, Chalco Mexico

\section{Email address:} \\ francisco.bulnes@tesch.edu.mx (F. Bulnes),kolob@yahoo.com (I. Martínez),omarzon_07@hotmail.com (O. Zamudio), \\ ing.gabrieltec@gmail.com (G. Negrete)
}

\section{To cite this article:}

Francisco Bulnes, Isaías Martínez, Omar Zamudio, Gabriel Negrete. Electronic Sensor Prototype to Detect and Measure Curvature Through Their Curvature Energy. Science Journal of Circuits, Systems and Signal Processing. Vol. 4, No. 5, 2015, pp. 41-54.

doi: $10.11648 /$ j.cssp.20150405.12

\begin{abstract}
Through of the concept of curvature energy, and the curvature theory on homogeneous spaces is designed and constructed an electronic sensor prototype to measure curvature in 2, and 3-dimensional spaces using the programming of shape operators in micro-controllers and the value of their energy integrals along the curves and geodesics in their principal directions. The curvature obtained under the sensor device is their spectral curvature given in voltage in 2-and 3-dimensions and their perception of curvature through electronic signals on a curved surface is the curvature energy that is detected in the displacement of an accelerometer on the curved surface.
\end{abstract}

Keywords: Curvature Energy, Energy Spectrum of Curvature, Gaussian Curvature, Principal Curvatures, Curvature Accelerometer Detection

\section{Introduction}

In the study of the field observables are of major importance from a point of view of their geometry, the curvature and torsion of a space affected for a field ${ }^{1}$, which are the geometrical invariant that defines their shape and orientation.

In our design analysis, we will consider like model of curvature, the obtained one by the Gaussian curvature, which involves from the mathematical point of view, curvatures along geodesic, curves on surfaces and space, sections of curved surfaces and bodies in the space, considering the design of an operator of shape based at the beginning in the geometry of the sphere $S^{2}$. Likewise, the value of certain integrals on cycles (invariant classes of the space $^{2}$ ) and

1For example the gravitational field that produces a geometrical scenery in the space-time affecting to this by the only presence of the matter. Likewise, their curvature is the field observable deduced by the curvature tensor of the corresponding field equation of Einstein.

2 For example, circles to the 2 - sphere, horocycles to a hyperbolic disc, planes to Euclidean space, etc., where can be evaluate the integrals of lines, surfaces, or considering the case of 2 - surfaces, on which is measured the curvature through the two principal directions representing these two values, as the maximum and minimal value of curvature of the surface determining their shape [1], can be established the curvature interval $k_{1} \leq k \leq k_{2}$, [1], on which we design the programming of our sensor. This obeys to a question of design of the sensor, and also of the space perception of the controller on the accelerometer device, which must to use a modulation space with a domineering energy condition given by [1-7]:

$$
[V]^{2} \int_{C} h k^{2} d s \geq\left(\int_{C} h^{2}-k\right)^{2} d s \geq \frac{1}{2} A V^{2} \int_{0}^{2 \pi} k(\theta) d \theta,
$$

where $V$, is the applied potential energy of curvature ${ }^{3}, \mathrm{~A}$,

curves. For example, the integrals $\int_{S^{1}} \omega$, on circles to a 2 - sphere.

3 This potential energy of curvature could be represented in an electronic applications as voltage. The integral expression is a censorship of curvature condition which is a Hilbert inequality as given in (1). 
is the area of the surface and $h$, their mean curvature and the last integral correspond to the curvature energy employed to measure the roundness in their displacement to along of two principal directions.

Through certain studies of the models of Gaussian and normal curvatures to determine by Hilbert inequality their curvature energy, we can on certain bound of roundness obtained through of the implementation of the spherical operator (spherizer $[7,8])^{4}$,

$$
\mathcal{O}_{E}=d(\gamma) \operatorname{Hom}_{K}\left(M, S^{2}\right) \int \Omega,
$$

use the idea of the Radon transform to determine through cycles and their co-cycles the curvature of the surface or body having the Hessian of the 2 - fundamental form $\Omega$, of the shape operator as a fundamental part of a censorship when we consider functions that are 2 -differentiable functions in the corresponding principal directions.

The development of research in theory of curvature in homogeneous spaces [8], has established that the measure of curvature can be obtained as an extrinsic curvature of the space classes (cycles) which have a curvature measure well defined. From a point of view of the signals and system analysis the utilization of these cycles could be translated in the context of the Fourier analysis in the application of energy pulses $\pi(x, y)$, [7] that can reproduce in the infinitum the measure the curvature through of their energy spectrum (see the figure 1).

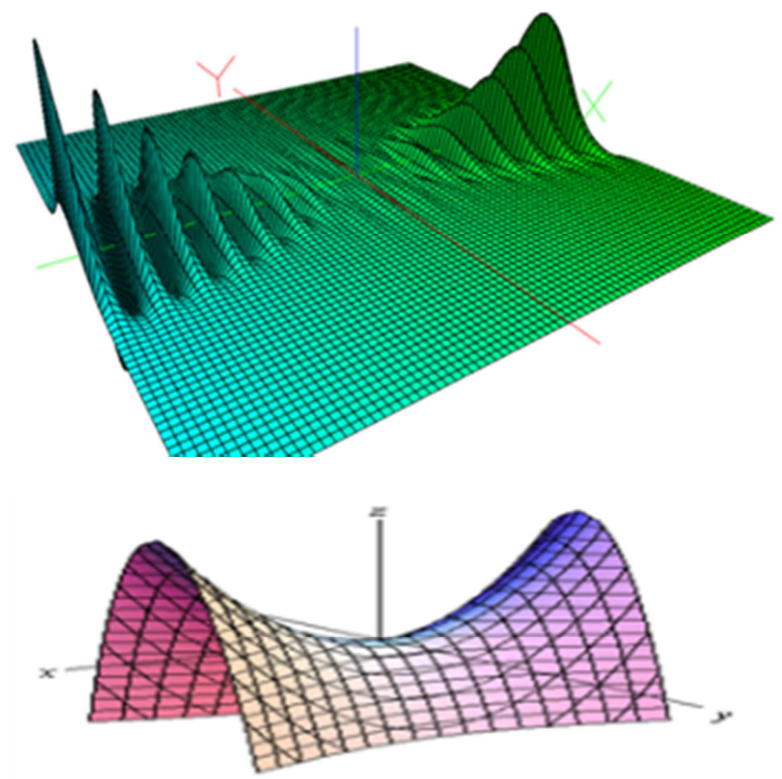

Fig. 1. The shape of a2-surface (hyperbolic paraboloid or "ride chair") is determined through energy Gaussian pulses [9]. Remember that the curvature is the more important geometrical invariant that determines the shape of a space.

4 Integral operator that involves a spherical map in the sphere $S^{2}$. The spherizer will be very useful in our design of sensor device as superior bound of the factor of gravity considered in the design of our accelerometer. Likewise, $\mathcal{\Theta}_{E}=2(-1)^{2} \times 1 \times(4 \pi)^{2}=8 \pi^{2}$.
These cycles must be invariant under translations and rotations ${ }^{5}$, to use them as symmetrical patrons in electronics and photonics of the energy signals that we want consignee in curvature information. Likewise, considering the curvature as a fundamental 2 - form whose representation in a Hilbert space (energy space) is given by $\Lambda_{g}(f) v$, $\forall v \in V(\zeta), \zeta \in K^{\wedge}$, and $f \in C_{c}^{\infty}(G / K),{ }^{6}$ (theorem (F. Bulnes) [8-10]) we have that:

$$
<\Lambda_{\tilde{g}}(f), f>=\bigoplus_{\zeta \in K^{\wedge}}<\Lambda_{\tilde{g}}(V(\zeta)), f>
$$

which is an "energy" representation of curvature. This permit us generalize the idea of the curvature as field observable to a level of their energy, having the concept of curvature energy, that is to say, the domineering energy in the action of the field on a curved space [11] to displace a particle on said space (see the figure 2).

5 The Gaussian pulses $z=e^{-x^{2}-y^{2}}$, are invariant under rotations and translations. Indeed, applying the Fourier transform we have:

$\mathscr{F}\left\{e^{-x^{2}-y^{2}}\right\}=\int_{-\infty}^{+\infty}\left\{\int_{-\infty}^{+\infty} e^{-x^{2}-y^{2}} e^{-j\left(\omega_{1} x+\omega_{2} y\right)} d x\right\} d y$

$=\int_{-\infty}^{+\infty}\left\{\int_{-\infty}^{+\infty} e^{-x^{2}-y^{2}+\left(j\left(\omega_{1} x+\omega_{2} y\right)\right)} d x\right\} d y$

$=\int_{-\infty}^{+\infty}\left\{\int_{-\infty}^{+\infty} e^{-\left(x^{2}+j \omega_{1} x\right)} e^{-\left(y^{2}+j \omega_{2} y\right)} d x\right\} d y$

$=\int_{-\infty}^{+\infty}\left\{e^{-\left(y^{2}+j \omega_{2} y\right)} \int_{-\infty}^{+\infty} e^{-\left(x^{2}+j \omega_{1} x\right)} d x\right\} d y$,

Completing the square in the argument of exp integrant, we have that the last expression of the double integral takes the form:

$$
\begin{aligned}
& \int_{-\infty}^{+\infty}\left\{\int_{-\infty}^{+\infty} e^{-\left(y^{2}+j \omega_{2} y\right)} \int_{-\infty}^{+\infty} e^{-\omega_{1}^{2} / 4} e^{-\left(x+\frac{j \omega_{1}}{2}\right)^{2}} d x\right\} d y \\
& =\int_{-\infty}^{+\infty} e^{-\left(y^{2}+j \omega_{2} y\right)}\left\{e^{-\omega_{1}^{2} / 4} \int_{-\infty}^{+\infty} e^{-(x+j \omega / 2)^{2}} d x\right\} d y \\
& =\int_{-\infty}^{+\infty} e^{-\left(y^{2}+j \omega_{2} y\right)}\left\{e^{-\omega_{1}^{2} / 4} \int_{-\infty}^{+\infty} e^{-u^{2}} d u\right\} d y, \\
& \text { If we call } x+\frac{j \omega}{2}=u, \text { then } d u=d x, \text { where } \\
& \int_{-\infty}^{+\infty} e^{-\left(y^{2}+j \omega_{2} y\right)}\left\{e^{-\omega_{1}^{2} / 4} \int_{-\infty}^{+\infty} e^{-u^{2}} d u\right\} d y=\sqrt{\pi} e^{-\omega_{1}^{2} / 4} \int_{-\infty}^{+\infty} e^{-\left(y^{2}+j \omega_{2} y\right)} d y,
\end{aligned}
$$

Newly, completing the square of the argument of $\exp \left\{-\left(y^{2}+j \omega_{2} y\right)\right\}$, we have that:

$$
\begin{aligned}
& \sqrt{\pi} e^{-\omega_{1}^{2} / 4} \int_{-\infty}^{+\infty} e^{-\left(y^{2}+j \omega_{2} y\right)} d y=\sqrt{\pi} e^{-\omega_{1}^{2} / 4} e^{-\omega_{2}^{2} / 4} \int_{-\infty}^{+\infty} e^{-w^{2}} d \omega \\
& =\pi e^{-\frac{1}{4}\left(\omega_{1}^{2}+\omega_{2}^{2}\right)},
\end{aligned}
$$

which is also a Gaussian pulse.

$6 \widetilde{g}$, is the pseudo-Riemannian metric in $G / K$, induced of the pseudo-Riemannian metric of the manifold $M$. 

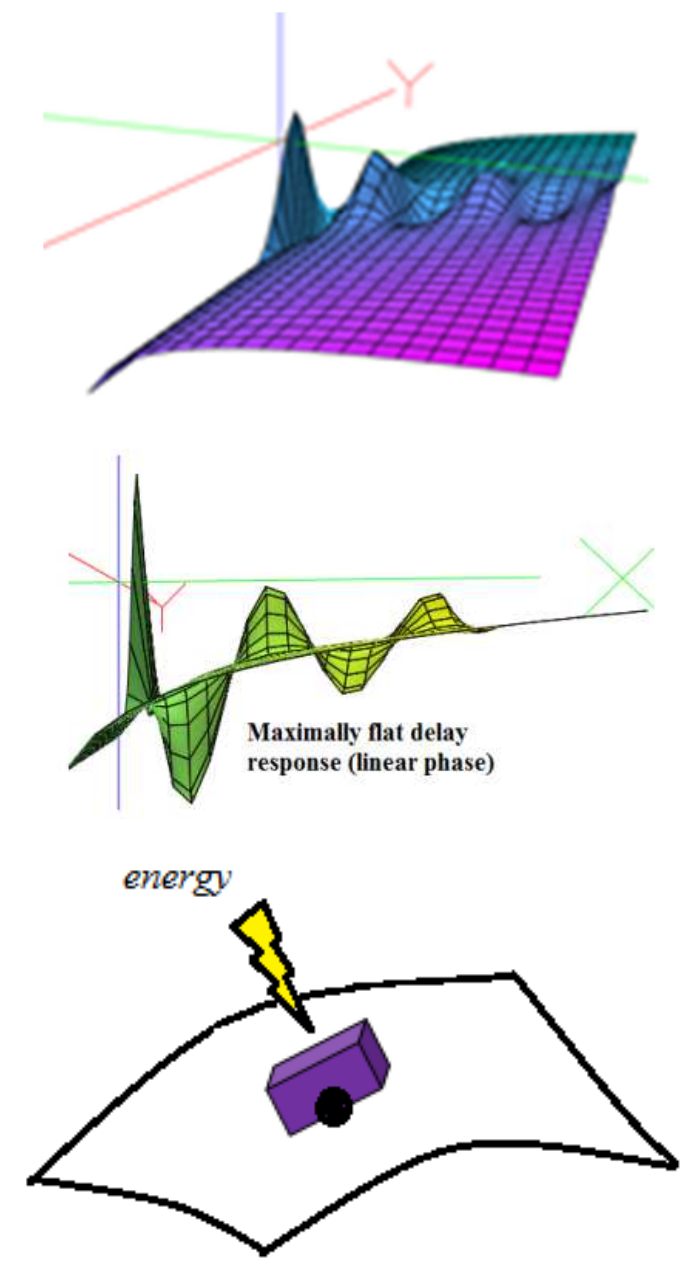

Fig. 2. The curvature energy is the unit of energy that a device perceives in their movement. The curvature is the spectrum of energy of the surface incurving.

Of fact, this idea is consigned, to the case of solid state electronics, in the first curvature sensor prototypes published in [7], with the application of the censorship given by (1) and followed after in the research of field curvature and torsion to quantum gravity [11-14] to the prospective of a sensor of quantum gravity. Also with this idea were designed other devices of photonics and opto-electronics nature considering light waves in the detection and measurement of curvature [15-17]. In this sense, is published a result with applications in opto-electronics and photonics in [17]:

Theorem (F. Bulnes) 1. 1. $[17,18]$. The Radon transform of the Gaussian curvature whose detection condition is the inequality (censorship ${ }^{7}$ ):

$$
[\log \phi(\xi(t))]^{2}\left[\int \log \sigma(t)\right]^{2} \geq\left(\int \Omega\left(1-\nabla^{2} \log \Omega\right)\right)^{2} \geq 4 \pi \int \Omega
$$

and using the signals the curvature measured by light beam, is:

7 Theoretical sensor of curvature in presence of the incurve and detected by a wave of light $[17,18]$.

$$
\iint\left|K\left(\phi, L_{\zeta}, \bullet\right)\right|_{L^{1}}=\frac{2}{R} \iint_{D^{2}}\left|K_{h}(\sigma(t))\right| d x d y
$$

Proof. $[17,18]$.

A design and construction of a sensor device needs the recognition on the part of the accelerometer of the property of roundness of a surface or body (see the figure 3), their perception of this roundness from the point of view of the signals of the sensor that the accelerometer will involve in their advance (displacement), and their aptitude to cause information in real-time, according to this perception as curvature of the surface or body (reading of the sensor). Then we want the following theorem [7]:

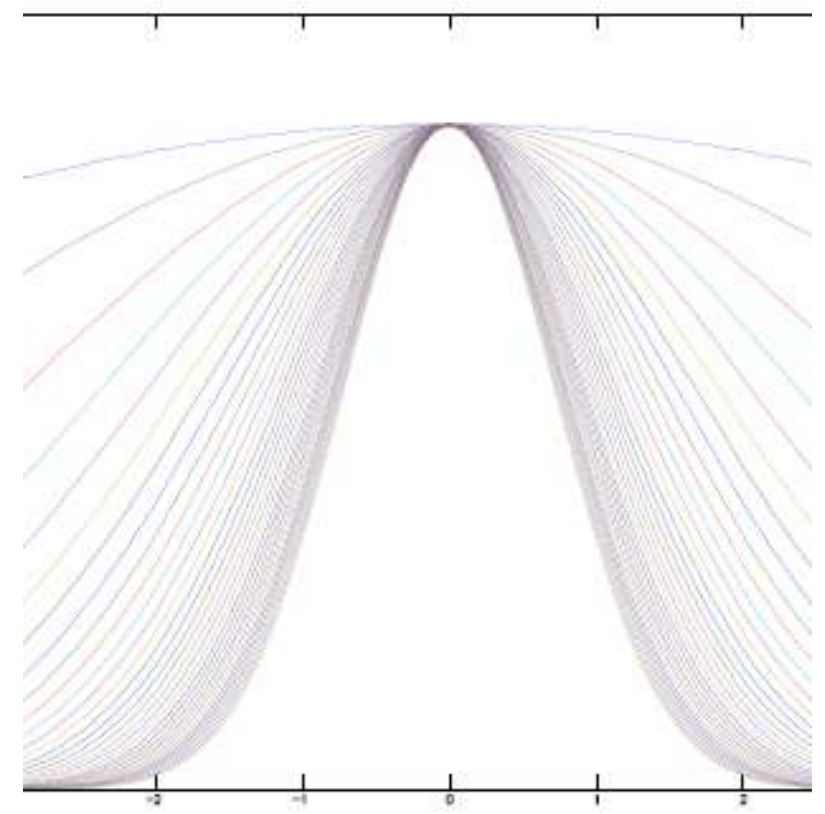

Fig. 3. Measuring curvature through their energy using Gaussian pulses as cycles. The different space curved parts can be measured using the adequate Gaussian pulse.

Proposition (F. Bulnes, I. Isaías, O. Zamudio, G. Negrete) 1. 1. To the 1 -dimensional image of a Gaussian pulse, the advance of the accelerometer must be proportional to the length from 0 to the intersection of the straight line of equation $\omega=p$ with the Gaussian pulse. Then an energy co-cycle (in the Radon space ${ }^{8}$ ) is that, whose energy area is:

$$
A(p)=2 \pi e^{-p^{2}}
$$

Proof. We consider the projection of the 2-dimensional Gaussian pulse $\pi\left(\omega_{1}, \omega_{2}\right)$, on the $\Omega_{1} \Omega_{2}$ - plane, that is to say, the pulse $\pi(\omega)=\pi e^{-\frac{1}{4} \omega^{2}}$. We consider a cycle given by the line $\omega=p$, intersecting the pulse as can see it in the

8 The Radon space is the space determined for the corresponding Radon transform of the object space where the special distribution of some physical property is defined. 
figure 4 . Then $\phi=0$. Then the co-took or co-cycle required is such that

$$
\pi(p, 0)=\frac{\partial A(p, \phi)}{\partial p},
$$

But the area $A(p)$, under de Gaussian pulse and the line $\omega=p$, comes given as:

$$
A(p)=\pi \int_{0}^{p} e^{-\frac{1}{4} x^{2}} d x=\pi \sqrt{\pi} \operatorname{erf}(p),
$$

But by (7) we have

$$
\frac{\partial}{\partial p} A(p, 0)=\pi \sqrt{\pi} \frac{\partial}{\partial p} \operatorname{erf}(p)=2 \pi e^{-p^{2}},
$$

where $2 \pi=L\left(C_{\varepsilon}\right)$, to a maximum circle $C_{\varepsilon}$, of the sphere of radius $\varepsilon=1$, [7] which bounds the length of arc of the Gaussian pulse in the measurement of curvature in the surface in one direction (see the figure 3 ).

Then considering the voltages of output of our accelerometer device as the produced by the displacements when is considered the length of perception in a Gaussian pulse in the space (given for the length $p$ ) we can to define the sensoring advance of the accelerometer on surface (see the figure 4).
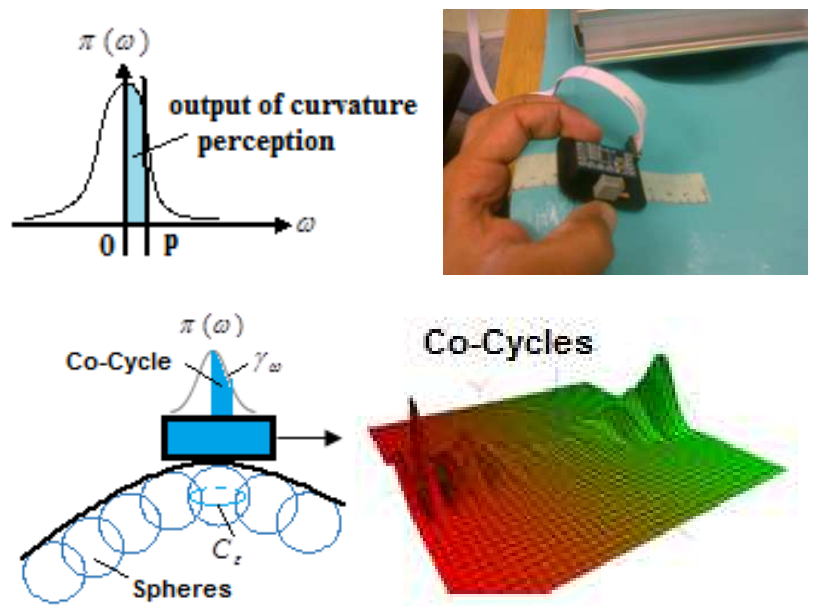

Fig. 4. Sensoring advance of the accelerometer on surface and their energy co-cycles.

\section{Development and Construction of the Electronic Sensor Prototype of Curvature. Optimization of the Curvature Sensor}

\subsection{Design and Fabrication of the Electronic Board of Micro-controller Pic16F877A Obeying the Gauging of Inter-phases with Accelerometer Actions}

As has been demonstrated in the theorem page 120, [7], the fabrication of our microcontroller must to be designed and gauged accord to the accelerometer actions, that is to say, the detected curvature energy data (given in voltages) must to be interpreted by the microcontroller in outputs of principal curvatures considering the two principal directions of the tangent space on surface that varies. The axis $Z$, must to be interpreted as $g$-cell factor in the monitoring action through cycles of energy (pulses) obtained when is varied the height in the displacement of our accelerometer (see the figure 5). Although also, in this $Z$-direction the output voltage in the sense strictly of the micro-controller is a principal curvature if we consider the theorem established in [7].

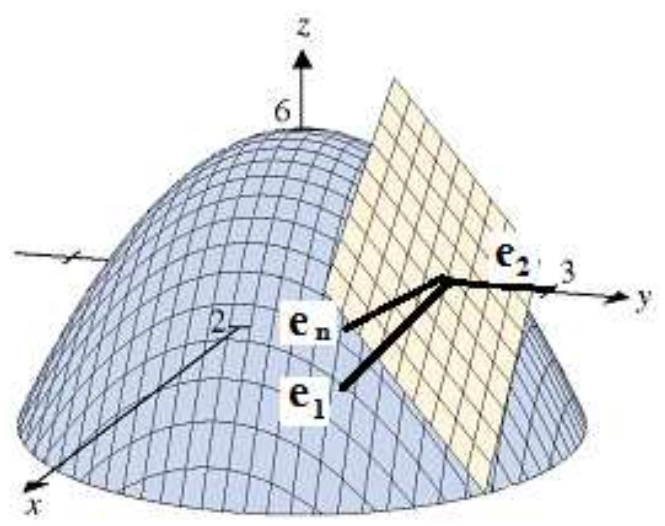

Fig. 5. Curved surface of equation $z=6-x^{2}-y^{2}$, and their tangent plane at point $(1,2,1)$. The principal curvatures $k_{1}$, and $k_{2}$, are measured in the canonical directions $e_{1}$, and $e_{2}$. Due to the symmetry of the surface, could be measured through principal curvatures in other directions $e_{n}$. This freedom will permit that our sensor can be displaced in any direction.

This design gives opportunity of to create an air version of this curvature sensor where can be used the Z-direction voltage output (see the figure 6). This variation of height can be considered as variation of the normal vector to the tangent plane, establishing through of the direction variation speed their normal curvature $k(u)$, where $k(u)=u S_{p}(u)$, where $u$, is an unitary tangent vector to a surface $M$ (see the figure 6). $S_{p}(u)$, is the shape operator depending the unitary normal field $U(p)$, in a neighborhood of $p \in M$. This will be of importance to define cycles of certain "length" inside of this neighborhood to the displacement perception of the accelerometer which will must to consignee spatial variations in curvature energy, this last in the micro-controller.

To this goal, were realized settings in the recorded programming in the micro-controller to the sensitivity of our accelerometer to that their displacement could be calibrated in the neighborhood established by the shape operator $S_{p}(u)$, and with it could be implemented the accelerometer sensored reaction our, with the displacement to can realize the measurements while our device is in movement. These settings consist in an electronic plate or board of micro-controller fabricated in laboratory considering a ceramic board of Siliceous with nodes aligned to constant distribution of charge annulling the decreasing of potential to 
electronic elements $[2-4,7]$.

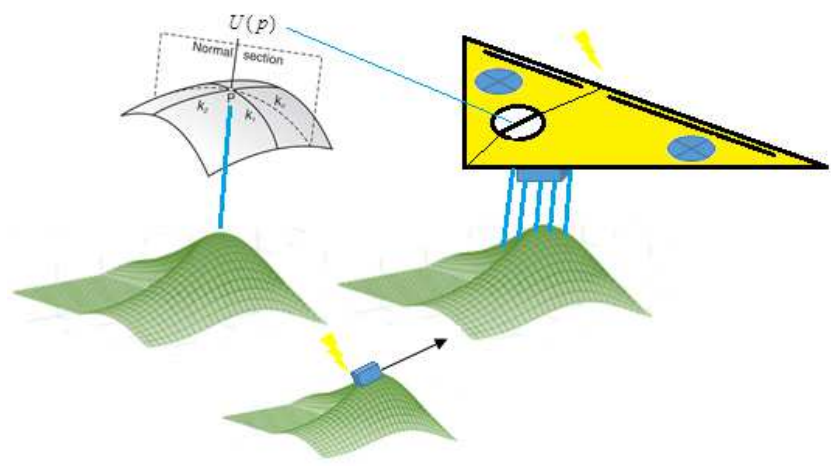

Fig. 6. Unitary normal field is reproduced by the height variation in the surface relieve by the air version of the curvature sensor, when the accelerometer is installed in a drone. In this case, we have that in a convex surface we have $U(p)=-N(0)$, then $k(u)<0$, and concave surface $U(p)=N(0)$, where $N(0)$, the reflected normal field is in the initial change of direction then $k(u)>0$, and detected by our sensor. In the case of terrestrial sensor, the field $U(p)$, is characterized by the gravity field.
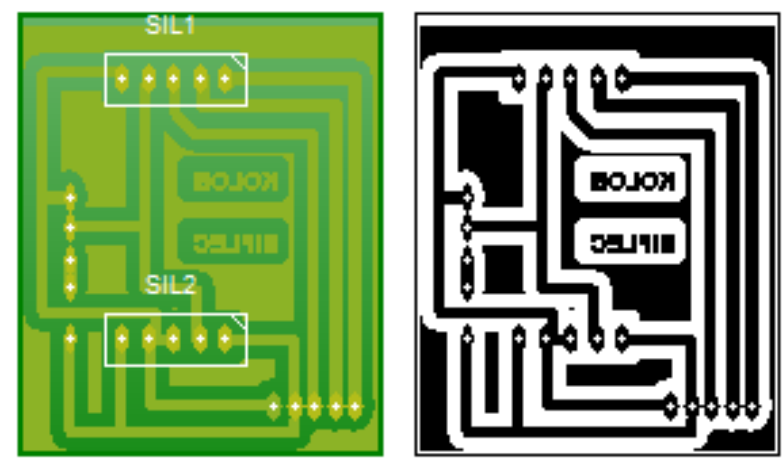

A)
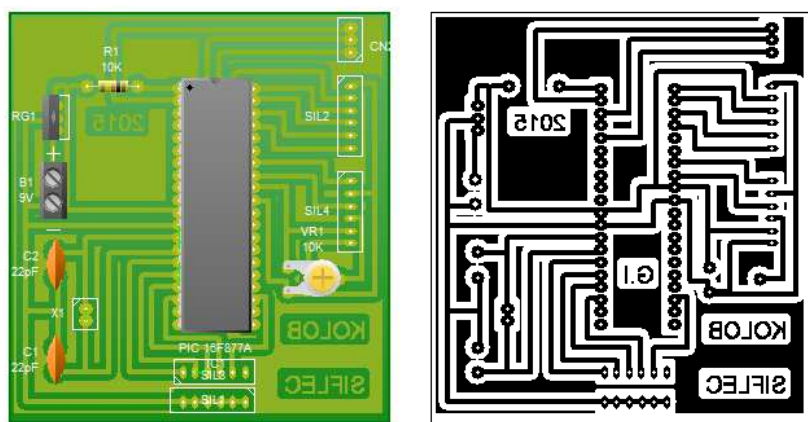

B)

Fig. 7. A). Design in PCB Wizard to printed circuit of the electronic board of the accelerometer MMA7361. B). Design in PCB Wizard to printed circuit of the electronic plate of the micro-controlled PIC16F877A, and feeding $5 \mathrm{~V}, \mathrm{CD}$ to the devices.

We need to design and construct an optimal electronic board in the accelerometer, whose voltage output data must be more exact and that responses with the displacement, not only when is in without movement (to future researches we want to implement an autonomous movement system of the accelerometer with certain velocity). To these goals in short and long terms, was modified the printed circuit to the electronic board, obtaining measurements in positive and negative voltages depending of the orientation and doing possible the exact measurements during their movement (see the figure 7) [15, 20, 21].

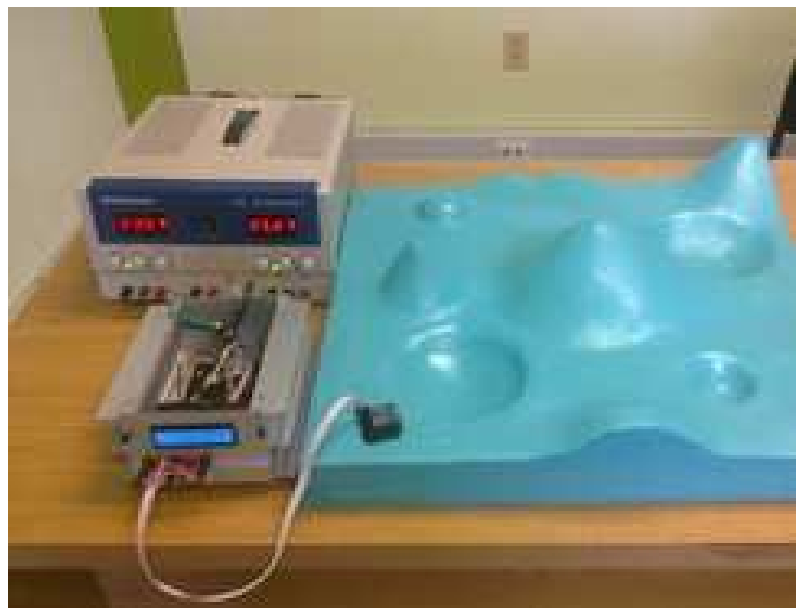

Fig. 8. Complete set of sensor curvature device of solid state.

\subsection{Displacement Perception Condition}

We have obtained a displacement perception condition of curvature given by the "curvature length" [7] (see the figure 9):

$$
\kappa=\operatorname{long}(k)=\sum_{0}^{N} r \sin \theta_{i}
$$

and that establishes a majoring condition of displacement to curvature sensor to the obtaining of mean measures of curvature in an energy space. Likewise, this majoring obeys to the result:

Proposition (F. Bulnes, I. Isaías, O. Zamudio, G. Negrete) 2. 1. The perception advances of curvature of the sensor though of the accelerometer must to satisfy to the sphere of radius $\varepsilon$, and sphered by operator $\Theta_{E}$, in a measured surface by the sensor that

$$
L(\gamma) \leq \kappa \leq L\left(C_{\varepsilon}\right)
$$

We can to apply the inequality $L\left(C_{\varepsilon}\right) \geq L(\gamma)$, [7] and establish that the superior extreme given in the inequality (11) is a condition of curvature energy of majored curvature given on a geodesic $\gamma$, of our cycle through their length ${ }^{9}$ (arc

9 Proof of the Proposition 2. 1. If we suppose that $\eta_{1}$, and $\eta_{2}$, are a pair sensor fields commuting in $M$ (that is to say $\eta_{1} \eta_{2}-\eta_{2} \eta_{1}=0$ [7]). Each of these fields generates a one-parameter group of diffeomorphisms in a neighborhood of a point on which is situated the accelerometer $z$.

If we consider the parallel transports $\tau_{t X}$, and $\tau_{t Y}$, respectively, along the flows of $\eta_{1}$, and $\eta_{2}$, for time $t$. Parallel transport of a vector $v \in T_{z} E$, around the quadrilateral with sides $t X, t Y,-t Y .-t X$, is given by $\tau_{s X}^{-1} \tau_{t Y}^{-1} \tau_{s X} \tau_{t Y} v$ 
This measures the failure of parallel transport to return $Z$ to its original position in the tangent space $T_{z} E$. That is to say there is "obstacle" in this surface that generates deviations. Shrinking the loop by sending $s, t \rightarrow 0$ (to two limits as (13)) gives the infinitesimal description of the following deviation, which finally define curvature [8]:

$$
\left.\frac{d}{d s} \frac{d}{d t} \tau_{s X}^{-1} \tau_{t Y}^{-1} \tau_{s X} \tau_{t Y} v\right|_{s=t=0}=
$$

$\left(\nabla_{\eta_{1}} \nabla_{\eta_{2}}-\nabla_{\eta_{2}} \nabla_{\eta_{1}}-\nabla_{\left[\eta_{1}, \eta_{2}\right]}\right) v=R\left(\eta_{1}, \eta_{2}\right) v$

where $R$, is the Riemann curvature tensor. This tensor belongs to the space $\Omega^{2} \wp$, where $\wp$, is the curvature perception zone equal to the tangent space $T_{z} E$.

For other way, given $v(\eta) \in T_{\eta} \wp$, in $M$, we consider the curved arcs of curves family $\gamma_{t}(s, \eta)$, with $a \leq t \leq b, \eta \in M$, and $s \in(-\lambda, \lambda)$, such that are satisfied the conditions of maximally in length $L(s) \geq L(\gamma)$. Then the length function required in our experimentation (see figure 9) $L(s), \forall s \in(-\lambda, \lambda)$, comply to this curved arcs family of the field sensor and using the fact $v(\eta)=\frac{\partial \gamma}{\partial s}\left(\eta_{0}, \eta_{a}\right)$, we have $d L(v)=\left(\frac{d}{d s} L\left(\gamma_{s}\right)\right)_{s=0}$, where $d L(v)$, is explicitly expressing the mean of $v$, and their covariant derivatives. This definition of $d L(v)$, has the advantages to be independent of the choice of the family $\gamma_{t}(s, \eta)$. We need to demonstrate that curve arcs $\gamma_{s}$, are geodesics, that is to say, the minimal trajectories in the space-time $M$, created by the sensor fields in $\gamma_{s}$. Thus must to comply the minimal energy consumption established in minimal energy principle. But $\gamma_{s}$, is geodesic if and only if $d L(v)=0$, $\forall v \in T_{z} E$. But this means that the geodesics belonging to $M$, (to the space in question) are precisely the critical points of $L$, on $E$. Using the Hessian of $L$, in a geodesic $\gamma_{s} \in E$, we have the index form to $v$, is [3]:

$I(v, v)=\left(\frac{d^{2}}{d s^{2}} L\left(\gamma_{S}\right)\right)_{S=0}$,

We consider the lifting of the mapping $(s, \eta) \mapsto \gamma_{s}$ to the mapping

$\gamma:[a, b] \times(-\lambda, \lambda) \rightarrow O(M)$,

where $O(M)$, is the bundle of orthonormal frames over $M$, such that $\pi(\gamma(s, \eta))=\gamma(t, s)$. Let $\eta_{1}$, and $\eta_{2}$, vector fields in the spherical curve arc $[a, b] \times(-\lambda, \lambda)$, defined by $\eta_{1}=\frac{\partial}{\partial s}, \quad \eta_{2}=\frac{\partial}{\partial t}$,

We consider the forms $\theta, \omega$, and $\Omega$, the canonical, connection and curvature forms on $O(M)$, respectively. Then we can define the forms $\theta^{*}, \omega^{*}$, and $\Omega^{*}$, on the rectangle by

$\theta^{*}=\gamma^{*}(\theta), \quad \omega^{*}=\gamma^{*}(\omega), \quad \Omega^{*}=\gamma^{*}(\Omega)$,

Then in particular if we consider the pullback corresponding to the canonical form $\theta$, we have by definition $[1,8]$

$\theta * v\left(\eta_{1}\right)=d \theta_{\eta}^{-1}\left(v\left(\theta\left(\eta_{1}\right)\right)\right)$

Defining the function

$F=\left\{\theta^{*} v\left(\eta_{1}\right), \theta^{*} v\left(\eta_{1}\right)\right\}^{1 / 2}$,

so that, at each $(t, s) \in[a, b] \times(-\lambda, \lambda), F(t, s)$, is the length of the vector $v_{\eta_{1}, t}^{(s)}$. Then the length $L\left(\gamma_{S}\right)$, of curve arc will be

$$
L\left(\gamma_{S}\right)=\int_{\left[\eta_{0}, \eta_{a}\right]} F(t, s) d s
$$

Then their variation is $v(\eta)$.

Remember that $p_{\mid}$, and $p_{2}$, are connected by minimal trajectories, and the functions $\mu: E \rightarrow P$, and $v: E \rightarrow P$, (where $P=\{p \in M \mid p=v(\eta)\}$ ) the two homotopic equivalences given in the diagrams of [1] where we can have $\phi_{*}: \pi(T, \mu(\eta)) \rightarrow \pi(S, \nu(\eta))$, piece of Gaussian pulse proposition 1. 1). Here $\kappa$, is curvature length implemented by (10) [7]. In the next section the sensor field $\eta$, (which has three enters as vector field $\left.\left(h_{1}(t), h_{2}(t), h_{3}(t)\right)\right)$, will be characterized by the transference function $h(t)$, calculated through capacitance voltages of the electronic characteristics of sensor in the three directions of the accelerometer.

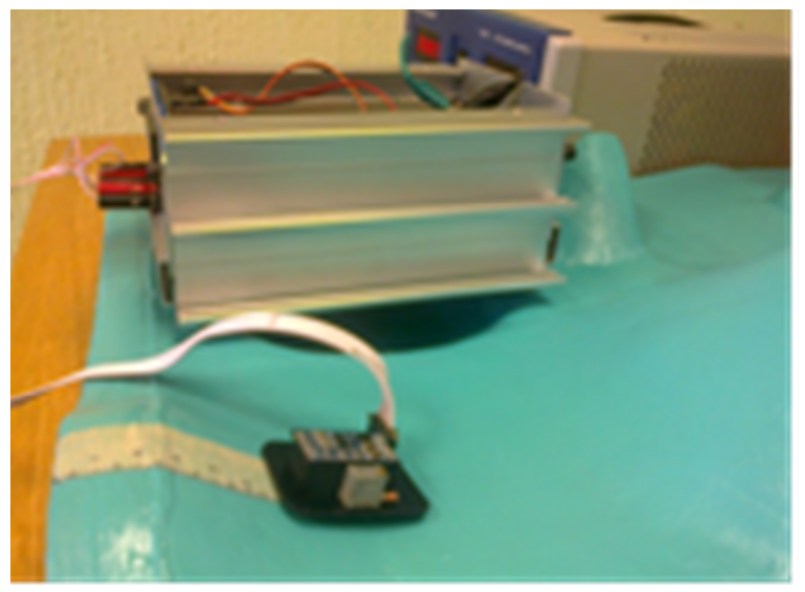

Fig. 9. Length function considered in our experimentation according (10) signed by adhesive tape.

The corresponding block diagram to $\varepsilon$ - data to the equation (10) is the following:

$$
\begin{aligned}
& \stackrel{r}{\square} \rightarrow \downarrow \rightarrow \square^{V\left(\theta_{1}\right)} \rightarrow \neg
\end{aligned}
$$

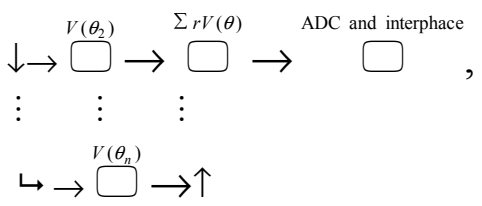

where the voltage outputs are given in the principal directions to a hypothetical accelerometer with sensor property in a $n$-dimensional space.

Then we can give the corresponding programming electronic design of the complete system of the microcontroller using the Master Prog recorder which was written in the Ram memory of the microcontroller PIC16F877A (see the figure 10).

which is an isomorphism. Then the variation of the length is given for $\delta\left(\int F(t, s) d s\right)=v(\eta)$. Then we re-written this integral through $\phi_{*}$, as: $\delta\left(\int_{[\mu(\eta), v(\eta)]}^{\left[\eta_{1}, \eta_{2}\right]} \phi_{*} F(t, s) d s\right)=\theta^{*}(v(\eta))$,

which proves that $L(\gamma) \leq \kappa$.

The other side of inequality (11) is proved under arguments of Gaussian and mean curvatures on surface using the same argument of length measures. 


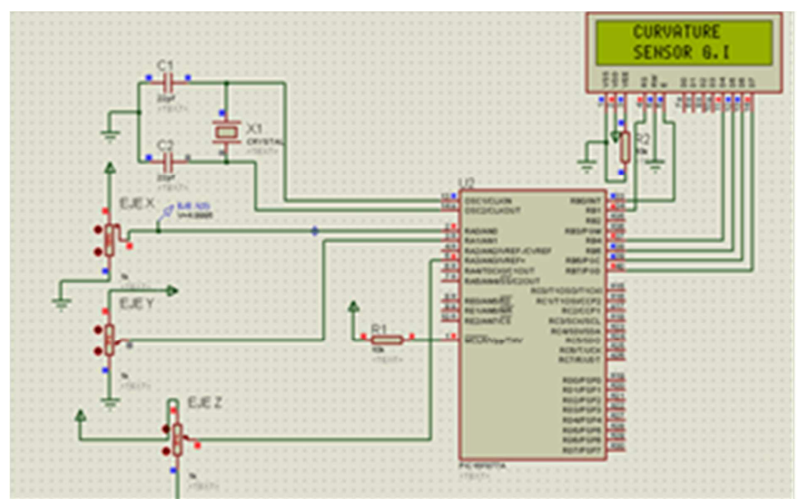

Fig. 10. Complete system of Microcontroller.

\subsection{Experiments}

The data will be observed and registered through of LCD, screen, verifying that the conversions in curvature energy are realized correctly in the outputs of the accelerometer $(x, y, z)$. The LCD, screen is divided of such form that are observed the conversion in the axis $X, Y$ and $Z$, the curvature energy in voltage outputs.

The electronic circuit of the curvature sensor was developed in proto-board to realize the simulating of physical manner and can to observe the analogic-digital conversion through a digital accelerometer MMA 7361, (with the pertinent modifications to detection of curvature) and the microchip microcontroller PIC16F877A (also with certain modifications to their optimal functioning). Also is added a LCD $18 X 2$ to viewing of the A/D conversion in a 3D-space (axis $X, Y$ and $Z$ ).

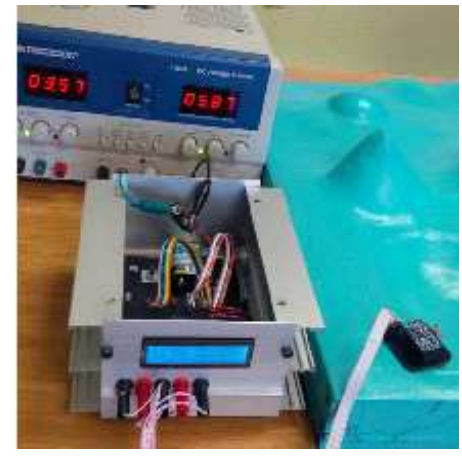

A)

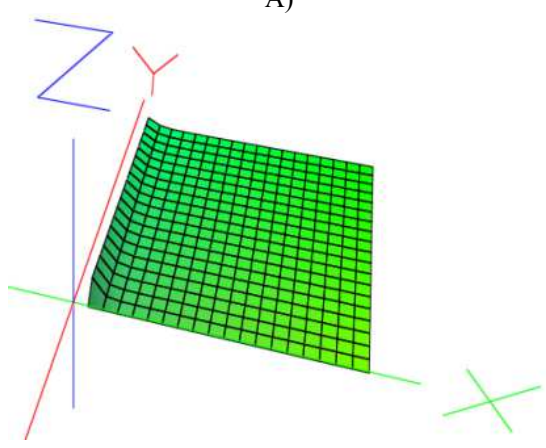

B)

Fig. 11. Initial position in a flat part of the experimentation 2-dimensional surface to the gauging of the sensor. B).
The first step is to observe the values through a flat surface are limiting to a $5 \mathrm{~V}$ conversion as maximum, to gauging effects of our sensing and their dependency of displacement (see the figure $11 \mathrm{~A})$ ). This produce us a sensitive platform in the sensor to obtaining of exact measures starting in a flat space which without curvature must to give 0 in their screen led (see the figure $11 \mathrm{~B}$ )). However, and due the physical conditions the sensor start with little deviation of order 0.0000025 (sensitivity at typical conditions divided for $1.5 \mathrm{~g}$-cell of the accelerometer at typical conditions).

We can to view the voltages in the three axis in different position in our LCD screen (see the figure 12).

The statistical data are considered to a short trajectory as showed in the figure 12 (see the values table I). The values increase in $X$-direction and $Z$-direction due to that the surface is concave. In the $Y$ - direction the curvature softy increases. The sensor perceive the curvature through the different values of voltage outputs in their displacement on a curved surface (see the table I, with the figure 13). Their mean curvature is soft elevation in oblique position.
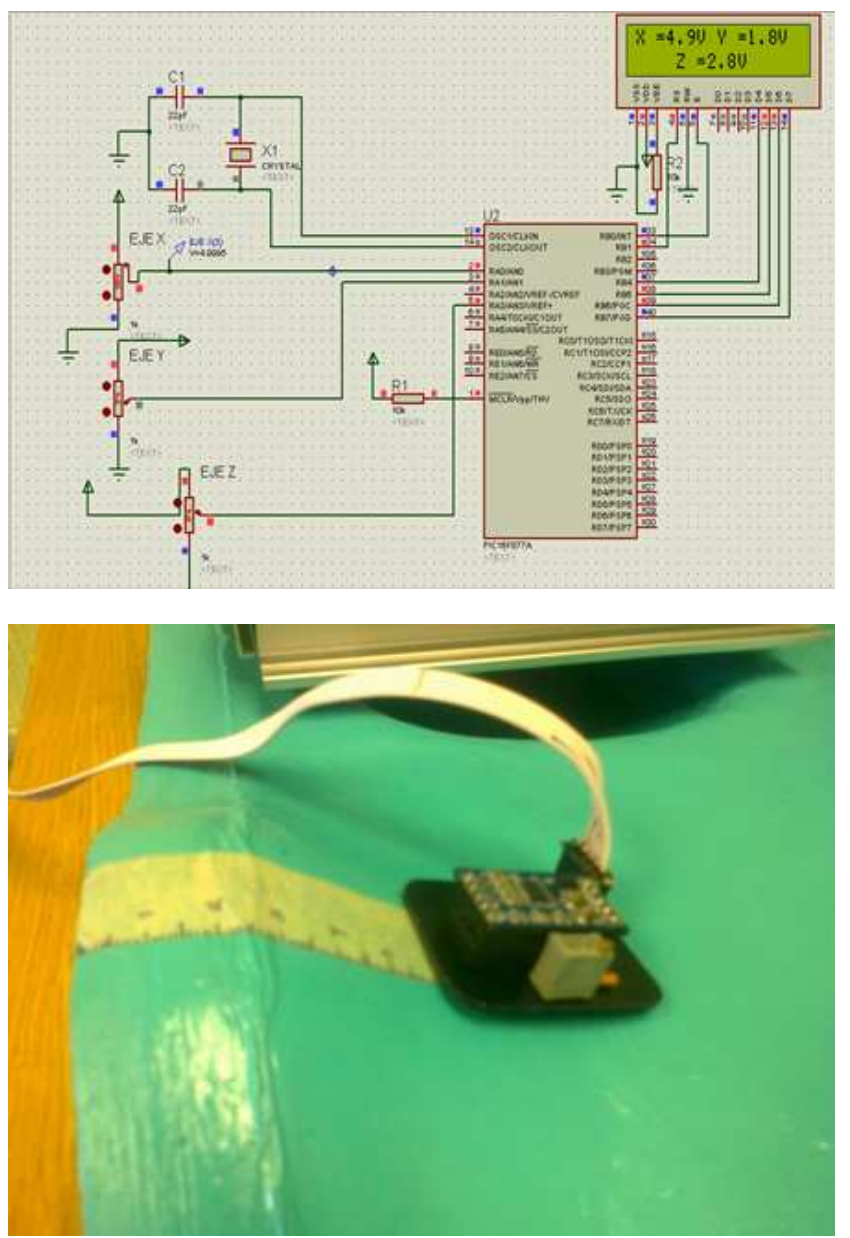

Fig. 12. Experimental proofs and rehearsals were realized in an amorphous surface in tha work table of the GI-TESCHA. 


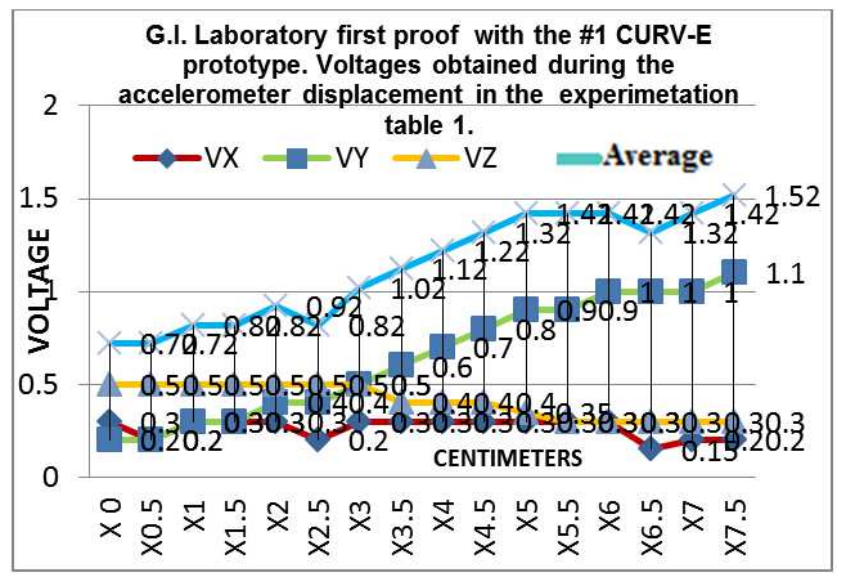

Fig. 13. Experimental sensing of curvature obtained through the voltage outputs to displacements in centimeters.

Table I. Experimental Measurements made by the curvature sensor device.

\begin{tabular}{lllll}
\hline Axis/Voltages & & & & \\
\hline Centimeters & $V_{X}$ & $V_{Y}$ & $V_{Z}$ & Average \\
\hline$X_{0}$ & 0.3 & 0.2 & 0.5 & 0.72 \\
$X_{0.5}$ & 0.2 & 0.2 & 0.5 & 0.72 \\
$X_{1}$ & 0.3 & 0.3 & 0.5 & 0.82 \\
$X_{1.5}$ & 0.3 & 0.3 & 0.5 & 0.82 \\
$X_{2}$ & 0.3 & 0.4 & 0.5 & 0.92 \\
$X_{2.5}$ & 0.3 & 0.4 & 0.5 & 0.82 \\
$X_{3}$ & 0.3 & 0.5 & 0.5 & 1.02 \\
$X_{3.5}$ & 0.3 & 0.6 & 0.4 & 1.12 \\
$X_{4}$ & 0.3 & 0.7 & 0.4 & 1.22 \\
$X_{4.5}$ & 0.3 & 0.8 & 0.4 & 1.32 \\
$X_{5}$ & 0.3 & 0.9 & 0.35 & 1.42 \\
$X_{5.5}$ & 0.3 & 0.9 & 0.3 & 1.42 \\
$X_{6}$ & 0.6 & 1 & 0.3 & 1.42 \\
$X_{6.5}$ & 0.15 & 1 & 0.3 & 1.32 \\
$X_{7}$ & 0.2 & 1 & 0.3 & 1.42 \\
$X_{7.5}$ & 0.2 & 1.1 & 0.3 & 1.52 \\
\hline${ }_{4}$ & & & &
\end{tabular}

Note: Proofs and rehearsals realized to $18^{\circ} \mathrm{C}$.

Mechanical Limitations: Size of the electronic board to measurements more precise, Design of basis to tangent vector.

During the displacement of the accelerometer in an amorphous surface is can to visualize in the led of the LCD, that the measures are realized of the speed and continuous form, we realizing of that the modifications in the configuration of the accelerometer MMA7361, are correct and efficient, thanks to the use of Gaussian pulses of high efficiency as has been demonstrated in the propositions 1. 1, and 2.1 .

\section{Spectral Curvature}

The detection and measurement of the curvature is realized using the value of the integrals of a field interacting on the geometric pattern along their surface doing it on signals of finite energy defined in the proposition 1. 1, and that they will code the information of curvature in a spectral space $\mathcal{L}\left(H\left(\Omega^{2}(M)\right)\right)$, (curvature energy) with $M$, a 2 dimensional space [20]; through of the signals given in the frequency established in our detector device that detects and measures curvature.

If $X_{s}$, is the electromagnetic field of EDMC/CURVE device (sensor field ${ }^{10}$ ) then we use the Radon transform such and as was mentioned in the theorem 1. 1, with the corresponding Hessian to calculate the curvature of a region $S$, of $M$. But this region is the energy region of the rays of light used to determine the measure of curvature with constant electromagnetic fields. This agrees with the Gaussian curvature, which use an identification of the sphere of dimension four [17] and solutions through of the use of the operator $L_{M A X}$, and that can be obtained by the formula [7]:

$$
\kappa=\int_{M}<A d(g) F_{D}, F_{D}>V o l_{g}=\int_{M} \operatorname{det} F_{D} V o l_{g},
$$

where $F_{D}$, is the corresponding Maxwell field required to the bundle of light of the connection $D$. To our very particular case (dimension two) our spectral Gaussian curvature will be:

$$
\kappa\left(\omega_{1}, \omega_{2}\right)=\mathscr{F}_{2}\left\{\mathfrak{h e s s} \mathcal{R}_{\sigma}(f(x, y))\right\},
$$

where to a Gaussian pulse $z=e^{-x^{2}-y^{2}}$, as cycle ${ }^{11}$, the Radon transform is

$$
\begin{aligned}
& \left.R_{\sigma}(f(x, y))\right\}=\int_{M} f(x, y) \delta(\pi(p, \phi)) d x d y \\
& =\int_{\Pi} f(x, y) d S(\pi(p, \phi))
\end{aligned}
$$

with $\quad d S(\pi(p, \phi))=\delta(\pi(p, \phi)) d\left(e^{-x^{2}-y^{2}}\right), \quad$ is the corresponding Dirac measure of the pulse defining their metric. Then their curvature measure by Gaussian pulses will be in the space:

$$
\begin{aligned}
& \kappa(p, \phi)=\mathfrak{h e s s s}\left.\mathcal{R}_{\sigma}(f(x, y))=\operatorname{hessif}(p, \phi)\right)\left(h_{1}, h_{2}\right) \\
&=1 / 2\left[h_{1}, h_{2}\right] \operatorname{det}\left(\begin{array}{ll}
\frac{\partial^{2} \hat{f}}{\partial p^{2}} & \frac{\partial^{2} \hat{f}}{\partial p \partial \phi} \\
\frac{\partial^{2} \hat{f}}{\partial \phi \partial p} & \frac{\partial^{2} \hat{f}}{\partial \phi^{2}}
\end{array}\right)\left[\begin{array}{l}
h_{1} \\
h_{2}
\end{array}\right],
\end{aligned}
$$

Considering the tempered distribution $[22,23] \hat{f}(p, \phi)$,

$10 X_{s}$, is a sensor field (field that measures the curvature through the manifesting if their energy to the detect said variations of changes of direction) then through the spectrum to obtain the measure as field observable taking the sensor field $X_{s}$, as reference.

$11 f(x, y)= \begin{cases}e^{-x^{2}-y^{2}}, & \text { if }|\mathrm{x}|,|y|<\frac{1}{2 \pi} \\ 0, & \text { if }|\mathrm{x}|,|y| \geq \frac{1}{2 \pi}\end{cases}$ 
we have ${ }^{12}$

$$
\kappa\left(\omega_{1}, \omega_{2}\right)=\int_{M} \kappa(p, \phi) e^{-j\left(\omega_{1} t_{1}+\omega_{2} t_{2}\right)} d p d \phi,
$$

which is our spectra of curvature to a measure realized by our accelerometer in an instant $t$.

What happen in the electronic characterizing of our sensor?

\section{Transitory Analysis of Response and Border Condition to $C$, and $V_{c}$, to the Co-Cycles of Curvature Energy}

We establish a linear model of response in voltage outputs of the sensor displacement as was explained in the sub-section 2. 2, which obeys to a differential equation of $R C$-type. We consider the following Kirchhoff law:

$$
V_{1}+V_{2}=V_{E}
$$

having that for each circuit element of the $R C$ - circuit with variable capacitor, the equivalent voltage is null:

$$
R \frac{d I}{d t}-\frac{I}{C}=0,
$$

The solution to the differential equation given by (19) is:

$$
I(t)=\lambda e^{\frac{1}{R C} t},
$$

The response (output) time of our accelerometer in the curvature sensoring is $\tau=2 m s \approx 0.2 c s$. We have a parameter depending of $R$, and $C$, to that relates the electronic part with their geometrical representation given by the Gaussian pulses that are co-cycles of curvature energy to conform our spectral curvature of the measured space $[7,2]$.

If our co-cycles are of the form $\frac{2}{\sqrt{\pi}} \alpha e^{-h\left(\omega_{1}{ }^{2}-\omega_{2}{ }^{2}\right)}$, then we need determine the parameter $h(R, C),[6]$ which must to come from a transitory analysis of response of our accelerometer ${ }^{13}$.

$12 \kappa\left(\omega_{1}, \omega_{2}\right)=\int_{M} \mathfrak{h} \mathfrak{e s s} \hat{f}(p, \phi) e^{-j\left(\omega_{1} t_{1}+\omega_{2} t_{2}\right)} d p d \phi$. Observe the analogy with the formula (13)

13 For other side, geometrically, the sensor relations between voltage outputs and displacement of the accelerometer in the space. Likewise if we consider a displacement in one direction, for example in the $X$ - axis, then could to have a differential equation of type:

$$
\frac{d V(x)}{d t}+x V(x)=V_{E},
$$

whose solutions in function of displacement are $V(x)=\frac{2}{\sqrt{\pi}} \int_{0}^{x} e^{-t^{2}} d t$. Then the

variation of this voltage respect to the displacement is $\frac{d}{d x} V(x)=\frac{2}{\sqrt{\pi}} e^{-x^{2}}$,

that is to say, the space is understanding through Gaussian pulses. Enters of the Hessian matrix must be curvature spectra of these pulses (see the figure 15).
We consider the differential equation to inputs and outputs system:

$$
V_{i n}=R C \frac{d V}{d t}+V_{C}
$$

where we have the transference function:

$$
H(s)=\frac{V_{C}(s)}{V_{\text {in }}(s)}=\frac{1}{R C s+1}
$$

Applying $\mathcal{L}^{-1}$, we have the dynamic solution of the system $\forall t \geq t_{0}$,

$$
h(t)=V_{i n} e^{-\left(\frac{1}{R C}\right) t},
$$

But considering that there exist a reference voltage $V_{\text {Ref }}=\beta \theta,{ }^{14}$ that affects the obtaining of voltage of the capacitance $C$, we have that $V_{\text {out }}=V_{\text {in }}-R C V=V_{\text {in }}-V_{c}$, where , is given by the system:

$$
V_{i n} \rightarrow \square \rightarrow V_{C}
$$

where

$$
h(t)=V_{\text {out }}=V_{\text {in }}-V_{C}=V\left(1-e^{-\left(\frac{\beta}{R C}\right) t}\right),
$$

which constitutes the electronic characterizing of the Gaussian pulse (co-cycle) having the $95 \%$ free of noise when is considered a pulse $\pi\left(\omega_{1}, \omega_{2}\right)=\frac{2}{\sqrt{\pi}} \alpha e^{-h\left(\omega_{1}{ }^{2}-\omega_{2}{ }^{2}\right)}$.

Table II. Additional Physical and Operating Characteristics of Our Accelerometer and Curvature Sensor.

\begin{tabular}{lll}
\hline Symbol & Physical Characteristic & Solutions and Ranges \\
\hline$V_{C}$ & $\begin{array}{l}\text { Transitory Voltage due to } \\
\text { Capacitance }\end{array}$ & $V_{C}=V_{\text {in }} e^{-\left(\frac{1}{R C}\right) t}$ \\
$V_{\text {in }}$ & Voltage Input & $V_{\text {in }}=V_{\text {out }}-V_{c}$ \\
$\tau$ & $\begin{array}{l}\text { Response Time of } \\
\text { Capacitance Voltage }\end{array}$ & $\begin{array}{l}\tau=R C \approx 0.002 s \\
C(V)=d Q(V) / d V\end{array}$ \\
$C$ & Capacitance & $C=\frac{1}{V_{C}} \int_{0}^{\tau} i(t) d t$ \\
$R$ & Resistance & $R=V_{\text {in }} / I$ \\
$\mathcal{O}_{E}$ & Spherical Sensitivity & $4 \pi \operatorname{dim} M$, \\
$Q$ & & $\mathcal{O}_{E}=2(-1)^{2} \times 1 \times\left(4 \pi^{2}\right)$ \\
\hline & Charge & $Q(t)=\alpha e^{\frac{1}{R C} t}+\frac{V_{E}}{R^{2} C} e^{\frac{1}{R C} t}$ \\
\hline
\end{tabular}

${ }^{14} \theta=\operatorname{Arctan}^{-1}\left(\frac{y}{x}\right)$ 


\begin{tabular}{lll}
\hline Symbol & Physical Characteristic & Solutions and Ranges \\
\hline$h$ & $\begin{array}{l}\text { Geometrical-Electronic } \\
\text { Parameter }\end{array}$ & $h(t)=\frac{1}{2 \pi j} \int_{-\infty}^{+\infty} H(s) e^{s t} d s$ \\
$\beta$ & $\begin{array}{l}\text { Parameter of Capacitance } \\
\text { Sensitivity to Curvature }\end{array}$ & $\begin{array}{l}\beta=V_{\text {Ref }} / \theta \\
0 \leq V \leq 5,\end{array}$ \\
$V_{\text {out }} V_{D D}$ & Output Voltage & $2.2 \leq V_{D D} \leq 3.6, \mathrm{~V}$, \\
$V_{\text {Ref }}$ & Reference Voltage & $V_{\text {out }}(x)=5 \operatorname{erf}(x)$ \\
$V_{\text {max }}$ & Maximum Voltage in & $V_{\max }=5$, \\
$\theta$ & operating & $\theta=A r c \tan ^{-1}\left(\frac{y}{x}\right)$ \\
$g$ & Angle of Deviation & \\
$V$ & gravity & $V=Q / C,-0.3$ to +3.6 \\
$T_{s t g}$ & Supply Voltage & -40 to $+125^{\circ} C$ \\
$S_{s}$ & Storage Temperature & with response time $\pm 0.002 \mathrm{~s}$ \\
$I$ & Supply Current & $400 \mu A \leq I \leq 600 \mu A$ \\
\hline
\end{tabular}

*From the differential equation $R \frac{d Q}{d t}+\frac{1}{C} Q=V_{E}$.
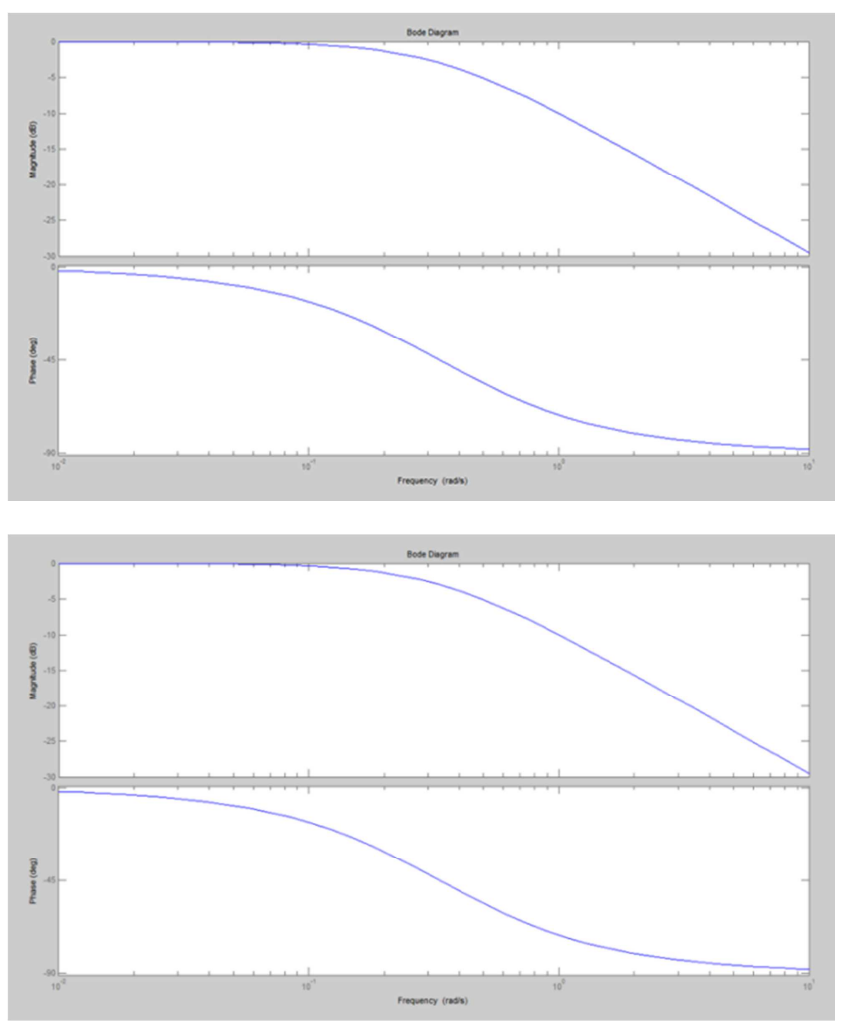

Fig. 14. Bode diagram of Band-with curves (phase and magnitude) of $V_{C}$, versus frequency the response analysis in frequency of our capacitance voltage. We observe that the noise in the device is very little. The used parameters are to the first transference function $>>n u m=1$; and to the second transference function are $>>$ den $=\left[\begin{array}{ll}3 & 1\end{array}\right] ;>>$ bode(num,den) num=1/3; >> den=[1 1/3]; >> bode(num,den).

Our curvature sensor operates in a range of voltage until $5 \mathrm{~V}$, permitting exact detections of curvature by the accelerometer with outputs given by $V(x)=5 \operatorname{erf}(x)$.

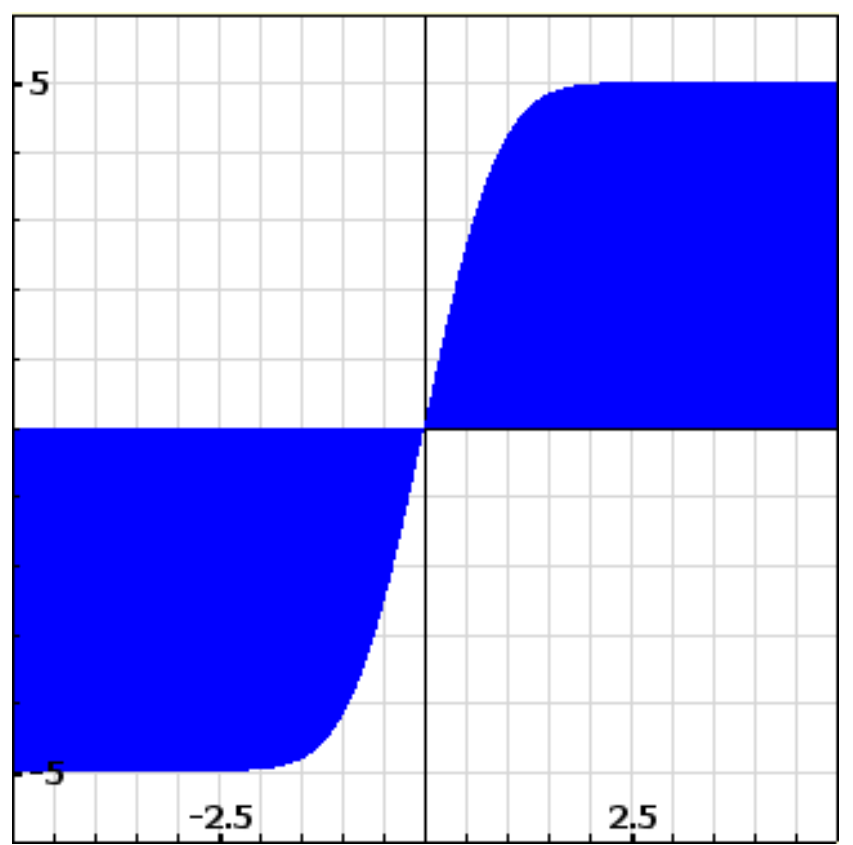

Fig. 15. Theoretical solution $V(x)=5 \operatorname{erf}(x)$. The negative part must be interpreted to space zones whose curvature is negative.

\section{Units}

We consider now, the diverse aspects of dimensionality of operating and physical operating conditions (see the table 2), as also approximations through non-harmonic analysis to the study the energy cycles using cylindrical functions in their re-construction. This last analysis helps to understand the interacting of physical space of the system and their cycles approximating the space, in this case the shape of the space. Likewise, considering the Fourier transform of the Gaussian pulse $\pi(x, y)$, to the obtaining in their spectral space (and with an appropriated polar transformation) we have:

$$
\widetilde{f}(\sigma, \tau)=\int_{0}^{\infty} r e^{-r^{2}} \int_{0}^{2 \pi} e^{\{-2 \pi i q r \cos (\theta-\varphi)\}} d \theta,
$$

with $\sigma=q \cos \varphi$, and $\tau=q \sin \varphi$. The integral over $\theta$, that is to say, which depends of $V_{\text {Ref }}$, which depends of capacitance $C$, is given by the function $2 \pi J_{0}(2 \pi q r)$, where $J_{0}$, is the Bessel function of order zero. The remaining integral of this Bessel function is a Hankel transform of order zero. Then (26) takes the form:

$$
\widetilde{f}(\sigma, \tau)=2 \pi \int_{0}^{\infty} r e^{-r^{2}} J_{0}(2 \pi q r) d r=\pi e^{-\pi^{2} q^{2}},
$$

In this step we have that the phase of our Gaussian pulse in the spectral space is a Bessel function, that to our sensor system we have $q=0.3$, and $r=0.2$. to the phase voltage $V_{\theta}$.

Finally involving the physical space, we have:

$$
\hat{f}(p, \xi)=\pi \int_{-\infty}^{\infty} e^{-\pi^{2} q^{2}} e^{i i 2 \pi q p} d q=\sqrt{\pi} e^{-p^{2}},
$$




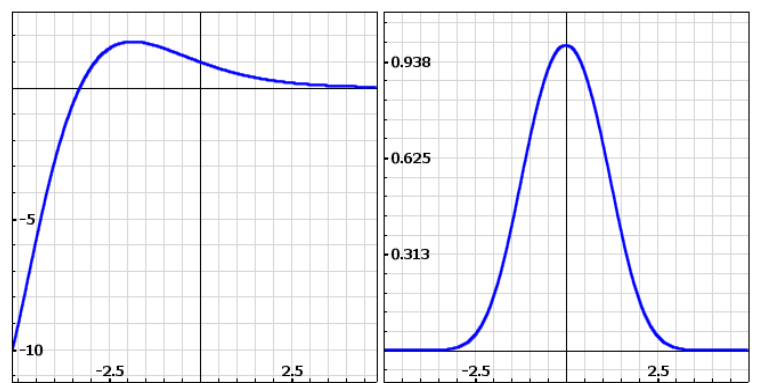

Fig. 16. Approximation through Bessel functions of the Gaussian pulses under regime of voltage produced by the capacitance $V_{\theta}=\operatorname{Bessel}(0.3 x, 0.2)$.

The physical properties of operating of our sensor function with the phase defined to our voltage $V_{C}$, given by Bessel functions that determine the variable modus of operating of the capacitor can be seen in the Table II. This phase involves the voltage $0.3 V_{D D}$, for one side and for other side $0.2 V_{\text {Ref }}$.

\section{Applications}

\subsection{Advanced Studies in Curvature as Field Observable: 2-Dimensional Curvature Model through Hyperbolic Waves}

An application to aero-spatial technology presented and published in the year 2002-2003 [18, 19], using the spectral model of curvature given in (17) according with the physical concept of curvature energy [7] to a 2-dimensional representation of curvature as tempered distribution of the space-time, using the 2-dimensional hyperbolic model of the space-time as hyperbolic disc and cycles given by hyperbolic waves is the spectral curvature:

$$
\begin{aligned}
& \kappa(\xi, p)=\frac{1}{2}\left\{( \mu b ) ^ { 2 } h _ { 1 } ^ { 2 } \left[\mathcal{U}^{\prime \prime \prime}\left(e^{\mu<z, b>}\right)^{2} e^{-\mu<z, a>} \widetilde{f}(\mu, b)+\right.\right. \\
& \left.+(\mu a)^{2} e^{-\mu<z, a>} \delta\left(e^{\mu<z, b>}\right) \widetilde{f}(\mu, b)\right]+(\mu z)^{2} h_{2}^{2}\left[\mathcal{U}^{\prime \prime \prime \times}\right. \\
& \left.\times\left(e^{\mu<z, b>}\right)^{2} e^{-\mu<z, a>} \widetilde{f}(\mu, b)\right]+(\mu a)^{2} e^{-\mu<z, a>} \delta\left(e^{\mu<z, b>}\right) \times \\
& \left.\left.\times(i b)^{2} \widetilde{f}(z, b)\right]\right\}+\left\{\frac{\partial^{2} f}{\partial \xi \partial b} h_{1}^{2}\left[\delta\left(e^{\mu<z, b>}\right) e^{-\mu<z, a>} \widetilde{f}(\mu, b)\right]\right\} \\
& +\left\{\frac{\partial^{2} f}{\partial b \partial \xi} h_{2}^{2}\left[\delta\left(e^{\mu<z, b>}\right) e^{-\mu<z, a>} \widetilde{f}(\mu, b)\right]\right\},
\end{aligned}
$$

where $\mathcal{U}$, is the step function in the horocycle, $\xi(z, b)$, $\widetilde{f}(\mu, b)$, is the hyperbolic Fourier transform in the same horocycle. The spectral curvature obtained in the plane (using the multi-physics modelling) comes given by the figures 17 and 18:

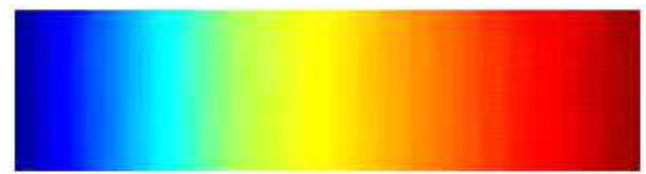

Fig. 17. Line spectra of curvature to the 2-dimensional hyperbolic model of space-time. Observe the long infra-red spectra according with the conclusions of the expansion of the Universe.

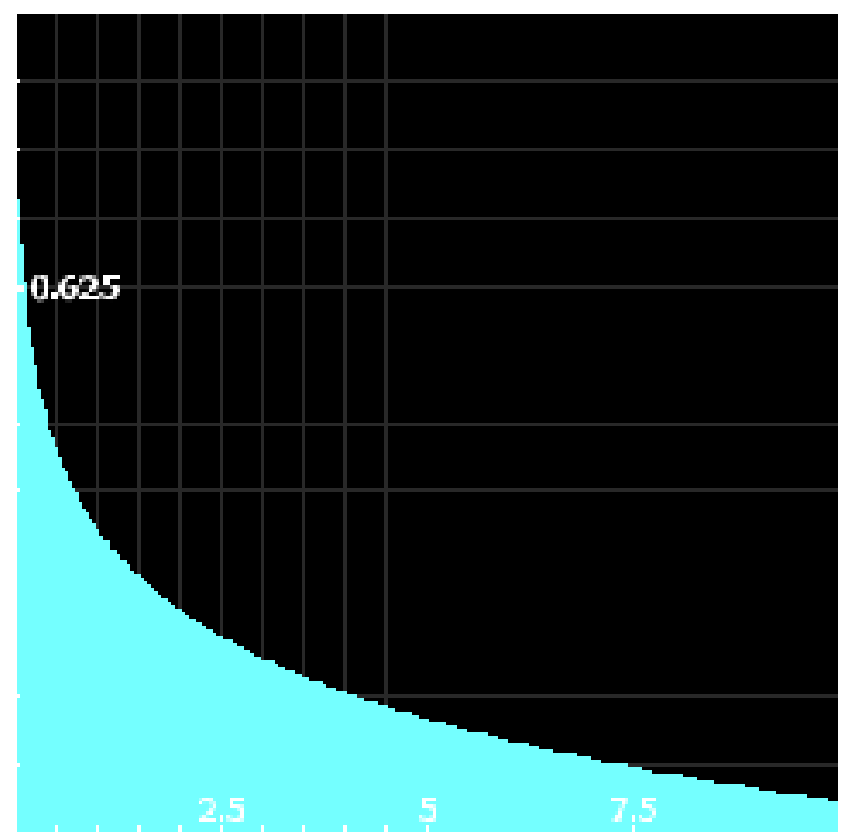

Fig. 18. The term $\exp \{-\mu<z, a>\}$, in the spectral curvature (29). This is proportional to the exponential decay given in the solution in the sensor device to the supply current $i=\frac{V}{R} \exp \{-t / R C\}$.

\subsection{Invisibility of Objects}

In certain transitory process of the light refraction effects produced for certain materials and their optics mechanisms the curvature can be fundamental to produce invisibility effects in an object, where certain curvature energy spilled in an object is diffracted, producing an effect of invisibility apparent, which is a deviation of the light that permits to observe the objects in their colors and details.

Of a more precise manage we can affirm that the effect is produced due to that the light field can be curved through of certain curvature energy that have the properties of curve the light, at least for the presence of some of the atoms of the certain materials that compose the object, producing an effect of curvature in the light, which generate an apparent invisibility (similar to like in the eclipses, although by distinct reasons, since in this last is due to the gravity).

\subsection{Applications to Fine Movements and Control of Drones}

The movement of drones to special mission are required with major precision and fine flight attitude of the drone in remote control can be optimized with the help of the curvature sensor which can establish in the drone a change of patterns of flight interpreting through curvature as smoothed movements and the object approximation as obstacle to the field sensor doing to vary the flux of their energy in their accelerometer.

A concrete application is to control a cup drone designed as helicopter to realize work of vigilance in a building in construction (see the figure 19). 

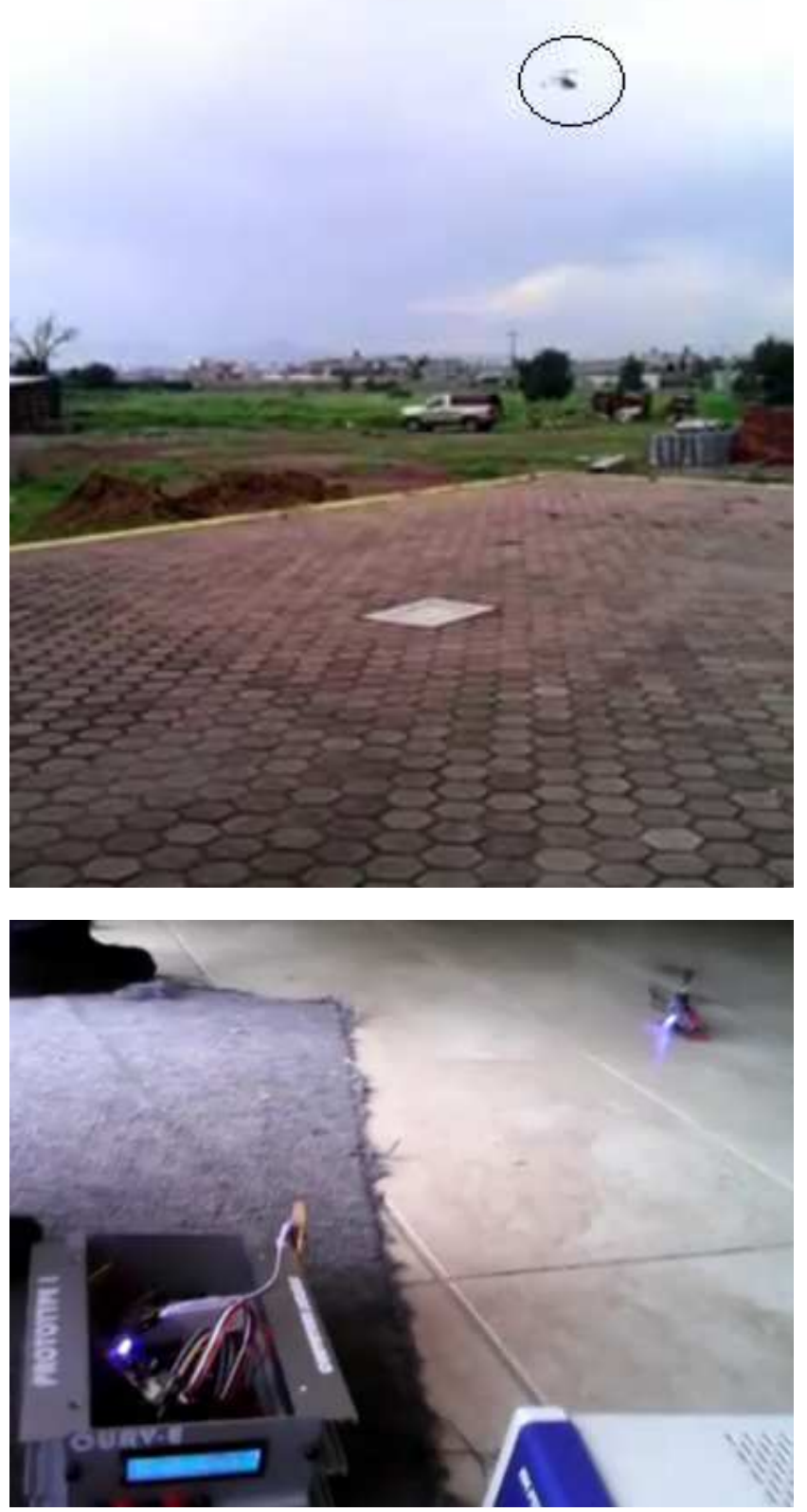

Fig. 19. Drone used to cup works in a building in construction where the drone has that to have a high precision of flight and quickly response in the monitoring of the building, likewise as capacity to recognize the material existence in the building.

\section{Conclusions}

The electronic prototype of solid state presented in this research has been developed through the curvature energy considering that in physical level, the perception of the curvature in the space has that to be given in terms of an electronic characteristic that has a relation with the geometrical enthrone where the direction change speed of the space due to the existence of curvature is established and perceived through of change of sensor field and their flux on the surface where is realized the measure. Considering that the Gaussian curvature can be approximated through the electronic pulses, these as special functions which are invariant under transformations of the space and their electrical characteristics, is constructed the curvature energy version of the Gaussian curvature and the mean curvatures using the principal curvatures as tempered functions whose spectra is an element of the space $\mathcal{L}\left(H\left(\Omega^{2}(M)\right)\right)$, where the transference function implicit to the system, $H(s)$, establish the relation of displacement with the system outputs in function of a $R C$ characterizing on the circuits where variable regime of capacitance will produce the sensor effect wanted. Then the voltage due the capacitance is $V_{C}=V_{0} e^{-\frac{1}{R C} t}$, with the bordering conditions

$$
\left\{\begin{array}{l}
C \rightarrow \infty, V_{C}=1 \\
C \rightarrow 0, V_{C}=0
\end{array}\right.
$$

These aspects can be majored under the use of certain semi-conductor materials that can to produce in the circuit of micro-controller low impedance to requirement of control in resonance by frequencies, for example in the accelerometer $[5,21]$. Also the requirement of current to the feeding of Micro-controller.

This prototype of curvature sensor is the first of many others that will be obtained under the same philosophy and principles on curvature energy where we want to establish a sensoring of the space through their energy, considering that the space and time are unified in an energy enveloping in all levels of measurement through their pure energy. Sub-sequence prototypes will be realized under electronics technologies versions with the goal of measure curvature and torsion as field observable and not as simple characteristics of the space. This last and after of many developments of sensor prototypes and as finally prototype will be designed under the same principle, curvature energy; a curvature and torsion sensor that has the work of measure the field interactions as particles that deform the space and time and to obtain a curvature sensor to detect and measure quantum gravity. To this case could be developed a SOIC-type circuit integrated in the micro-controller.

In the way, the applications will be much and diverse, for example, applications in MEMS developments and nano-materials, non-symmetrical fields technology, particles interferometers, special mimetic and optical effects devices, optimal control of drones, distributed generation considering the curvature to the design of special surfaces and tunnels, etc; and new metrologies.

Also we could to be obtained, under this same philosophy, applications in medicine, since curvature energy could establish deviations of energy, puddles or jumps which can be re-interpreted under spectral curvature as sickness in the body. To it, we will need curvature sensors that measure change of energy under field observables.

\section{Acknowledgment}

We are very grateful with Edgar Daniel Sánchez Balderas, 
Law B, Principal of TESCHA, Evaristo Vázquez, Hernández, LC, B, Financial Sub-principal, and Rene, Electronic Eng., Electronic Division Chief, by their material support and facilities to realize this research.

\section{Technical Notation}

$K$ - Curvature as general concept of roundness property. Also used in the paper as Gaussian curvature in a point $p$.

$k$ - Gaussian curvature along of the geodesic or surface.

$\mathcal{O}_{E}-$ Spherical operator which is related with the spherical map.

$k_{i}$ - Principal curvature in the principal ith-direction. In our research $\mathrm{i}=1,2$, only.

$V$ - Voltage.

$A-$ Area.

$\mathcal{L}\left(H\left(\Omega^{2}(M)\right)\right)$ - Space of spectral transformations on curvature $2-$ forms given in space $\Omega^{2}(M)$

$M$ - Space whose curvature is measured. In our study $M$, represent 2 -dimensional surfaces or 3 -dimensional bodies.

$S^{2}-2-$ Dimensional sphere. Also is the 2dimensional sphere used in the spherical map to design our curvature sensor

$r(P)$ - Evaluation of curvature radius from the product from their inverse principal curvatures.

$\Omega$ - Curvature form in the 4 , and 4 - dimensional spaces

$J_{1-r, p}-$ Bessel function of first specie.

$\mathscr{F}-$ Fourier transform.

$Y_{1-r, 1-r}-$ Bessel function of second specie.

SOIC - Small-outline integrated circuit.

$E D M C$ - Electromagnetic device to measure curvature

$\kappa-$ Curvature as value.

$\kappa_{G}-$ Gaussian curvature in the sense of the value of their integral.

$\kappa(p, \phi)-$ Spectral curvature in the Radon space.

$R$ - Radon transform.

$J_{0}$ - Bessel function of Zero order.

$\mathcal{L}$ - Laplace Operator of Laplace transform.

$\kappa\left(\omega_{1}, \omega_{2}\right)-$ Spectral curvature in the Fourier space.

$M M A-$ Aceelerometer type $M M$.

$P I C$ - Micro-controller type $P I$.

$P I$ - The PIC are a micro-controllers family of RISC type made by Microchip Technology Inc. and derived from PIC1650, originally developed by the micro-electronic technological firms. We use a PIC16F877A .

$M M$ - Micro-Machined. Free-scale Semiconductor's micro-machined accelerometer (MMA) series acceleration sensors are designed for end products or embedded systems that require measurement of forces resulting from tilt, motion, positioning, shock or vibration.

$L E D$ - Light Emitting Diode.
$L C D$ - Liquid Crystal Display.

$M E M S$ - Micro-electromechanical systems.

$S O I C$ - Integrated sensor Micro-circuit.

$P C B$ Wizard - Program designed to create electronic circuits to the after the design print circuits to one or two faces.

\section{References}

[1] Kobayashi, K. Nomizu, "Foundations of Differential Geometry”, Wiley and Sons, New York, 1969.

[2] V. Kaajakari, "Closed Form Expressions for RF MEMS Switch Actuation and Release Time," Electronics Letters, Vol. 43, No. 3, 2009, pp149-150.

[3] V. Kaajakari, A. T. Alastalo, T. Mattila, "Electrostatic Transducers for Micromechanical Resonators: Free Space and Solid Dielectric," IEEE Transactions on Ultrasonics, Ferroelectrics, and Frequency Control, Vol. 53, No. 12, 2006, pp. 2484-2489.

[4] O. Holmgen, K. Kokkonen, T. Veijola, T. Mattila, V. Kaajakari, A. Oja, J. V. Knnuttila, M. Kaivola, "Analysis of Vibration Modes in a Micromechanical Square Plate Resonator," Journal of Micromechanics and Microengineering, Vol. 19, No. 1, 2009, Article ID: 015028.

[5] V. Kaajakari, A. Lal, "Micromachined Ultrasonic Motor Based on Parametric Polycrystalline Silicon Plate Excitation," Sensors and Actuators A: Physical, Vol. 137, No. 1, 2007, pp120-128. Doi:10.1016/j-sna.20.

[6] A. T. Alastalo, V. Kaajakari, "Intermodulation in Capacitively Coupled Microelectromechanical Filters," IEEE Electron Device Letters, Vol. 26, 2005, pp189-191.

[7] Bulnes, F. Martínez, I, Mendoza, A. Landa, M., "Design and Development of an Electronic Sensor to Detect and Measure Curvature of Spaces Using Curvature Energy," Journal of Sensor Technology, 2012, 2, pp116-126. http://dx.doi.org/10.4236/jst.2012.23017.

[8] Bulnes, F., Research on Curvature of Homogeneous Spaces, TESCHA, Mexico, 2010, pp. 44-66. http://www.magnamatematica.org.

[9] F. Bulnes, "Curvature Spectrum to 2-Dimensional Flat and Hyperbolic Spaces through Integral Transforms," Journal of Mathematics, Vol. 1. No. 1, pp17-24.

[10] F. Bulnes, Integral Theory of the Universe, Proc. Applied Math 2, IMUNAM, ESIME-IPN, Mexico City, 2006, pp73-122.

[11] F. Bulnes, "Quantum Gravity Sensor by Curvature Energy: their Encoding and Computational Models, "IEEE Proc., SAI 2014, London, UK, 2014, pp. 855-861.

[12] Bulnes, F. "Electromagnetic Gauges and Maxwell Lagrangians Applied to the Determination of Curvature in the Space-Time and their Applications," Journal of Electromagnetic Analysis and Applications, Vol. 4 No. 6, 2012, pp. 252-266. doi: 10.4236/jemaa. 2012.46035.

[13] E. Guivenchy, "Torsion Matter and their Corresponding Dirac Equations on Neutrinos and Matter/anti-Matter Asymmetries (Part I: scattering difference between Neutrinos/anti-Neutrinos)," Journal on Photonics and Spintronics, Vol 3, no. 4, 2014, pp9-18. 
[14] S. Gorbuix, E. Guivenchy, "Torsion Matter and their Corresponding Dirac Equations on Neutrinos and Matter/anti-Matter Asymmetries (Part II: gravitation insights)" Journal on Photonics and Spintronics, Vol. 4 No. 1, pp15-25.

[15] http://www.mouser.com/ProductDetail/Silicon-Labs/EFM8BB 10F2G-A-QFN20/?qs=sGAEpiMZZMu9ReDVvI6ax9sqO0qr XIDW4ZuhKenb2c\%252bQvyUXU1UbuQ\%3d\%3d.

[16] Heath, S. (2003). Embedded systems design. EDN series for design engineers (2ed.)., Elsevier Science, Oxford, UK.

[17] F. Bulnes, E. Hernandez and J. Maya, "Design of Measurement and Detection Devices of Curvature through of the Synergic Integral Operators of the Mechanics on Light Waves," Proceedings of International Mechanics Engineering Conference and Exposition, Orlando Florida, 16 November 2009, pp. 91-102. doi: 10.1115/IMECE2009-10038.
[18] F. Bulnes, "Radon Transform and Curvature of an Universe," UNAM Postgraduate Thesis, 2001.

[19] F. Bulnes, Detection of finite energy signals to the measurement of the Universe curvature, Iberomet VII, Cancún, Quintana Roo, Mexico, 2002, pp825-843.

[20] L. J. Van Vliet, P. W. Verbeek, "Curvature and Bending Energy in Digitized 2D and 3D Images", Proceedings of 8th Scandinavian Conference on Image Analysis, Tromso, 25-28 May 1993, pp. 1403-1410.

[21] B. Nashelky, "Electronics: Circuit Theory and Electronic Devices," Pearson Prentice Hall, Upper Saddle River, 2003.

[22] W. Rudin, Functional Analysis, McGraw-Hill, Inc., USA, 1973.

[23] J. E. Marsden, A. J. Tromba, Vectorial Calculus, Addison Wesley, 1991. 